\title{
Traffic Management for Drones Flying in the City
}

\author{
Busyairah Syd Ali \\ Department of Software Engineering \\ Faculty of Computer Science \& Information Technology \\ University of Malaya \\ 50603 Kuala Lumpur, Malaysia \\ busyairah@um.edu.my
}

\begin{abstract}
Air Traffic Management (ATM) is designed based on the notion of a pilot navigating an aircraft from within the aircraft. Increasing demand for Unmanned Aircraft Systems (UAS) usage and its safe integration into segregated/non-segregated airspace, on the other hand, have raised a question on the adoption of the current ATM for the UAS Traffic Management (UTM). The Procedures for Air Navigation Services-Air Traffic Management (PAN-ATM) and Procedures for Air Navigation Services - aircraft operations (PAN-OPS) rules are both developed for manned flight operations. Therefore, this work (i) defines the UTM system and describes its envisioned functionalities; (ii) performs an exploratory research to identify the distinctions between a manned and an unmanned flight operations and consequently the possible challenges to implement UTM with regard to the ATM; (iii) determines potential Communication, Navigation and Surveillance (CNS) technologies to support the UTM system; and proposes an architectural framework for UTM based on the findings. It is important to highlight that this work is developed based on the assumption that the UAS is remotely - piloted and operates in the class $\mathrm{G}$ airspace ( 500 feet and below) in urban areas where the UAS are exposed to various obstacles such as static objects: high-rise buildings, trees, lamp posts, over-ground rail tracks and dynamic objects: cranes.
\end{abstract}

Keywords: UAS, UAV, UTM, ATM

\section{Introduction}

An unmanned aerial vehicle (UAV) is defined as a pilotless aircraft which is flown without a pilot-incommand on-board and is either remotely fully controlled (from ground, another aircraft or space) or programmed and fully autonomous (International Civil Aviation Organization, 2005). The UAV is also known as unmanned aircraft system (UAS) or more famously as drone. In this paper, all terms will be used interchangeably.

The increasing demand for UAS civilian applications include precision agriculture, fishery protection, package delivery, infrastructure monitoring, aerial photography and video, land surveying, environmental assessment, security surveillance, emergency response for medical services, forest fire detection, search and rescue, contamination measurement, recreation and many more applications to emerge in the near future. The large number of potential applications indicate the unmanned aircraft systems (sUAS) occupancy coverage of the airspace and hence the need for sUAS traffic management (UTM). In other words, an ATM like system is needed to safely and efficiently manage the sUAS traffic in a particular airspace. The idea of UTM was initially proposed at a recent National Aeronautics and Space Administration (NASA) convention. Since then, many researchers from both academia and industry have begun to investigate, develop and test potential methods and technologies for the UTM concept.

According to Jiang, T. et al.(2017), the UTM system will borrow fundamental ideas from the ATM despite the differences in method of control, maneuvering, function, range and operational constraints. Even though the field of research is still in infancy, a number of researchers have proposed potential 
concept of operation and system architecture for UTM. These include (Foina, Krainer, \& Sengupta, 2015; Jiang et al., 2017; P. Kopardekar, Rios, Prevot, Johnson, \& Jung, 2016; P. H. Kopardekar, 2017; Low, Gan, \& Mao, 2014; Pathiyil, Low, Soon, \& Mao, 2016; Prevot, Homola, \& Mercer, 2016; Prevot, Rios, et al., 2016; Ren et al., 2017).

In addition, the industry such as Amazon and Google have advanced in the UTM practice. Amazon (Amazon Prime Air, 2015a, 2015b) proposed an idea of “Determining Safe Access with a Best-equipped, Best-served Model for sUAS", which classifies the sUAS into categories based on vehicle equipment and technology, often referred to as equipage. Google's architecture of UAS Airspace System (Google Inc., 2015) also partitioned the airspace into several classes in accordance with Federal Aviation Administration's (FAA) definition.

All of the current works propose system architectures or models for UTM, comprising potential components of UTM and the data flow between the components. The architectures vary in term of their complexity. In addition, some of the researchers proposes UTM operational concepts. However, none of the work addressed potential Communication, Navigation, and Surveillance (CNS) means to support the UTM. It is a well-known fact that the CNS is the backbone of ATM.

This work (i) defines the UTM system and describes its envisioned functionalities; (ii) performs an exploratory research to identify the distinctions between a manned and an unmanned flight operations and consequently the possible challenges to implement UTM with regard to the ATM; (iii) determines potential Communication, Navigation and Surveillance (CNS) technologies to support the UTM system; and proposes an architectural framework for UTM based on the findings.

\section{UAS Traffic Management System (UTM)}

Kopardekar (2016) defined UAS Traffic Management System (UTM) as a research software application prototype that aims to safely and efficiently enable UAS operations in the low altitude airspace (class $G$ airspace) by allowing UAS operators to submit flight plans to execute a specific task; determining how to safely enable single or multiple UAS operations either within visual line-of-sight (VLOS) or beyond visual line-of-sight (BVLOS); and coordinating airspace services across many operators. Class $G$ airspace is typically the airspace very near to the ground (1,200 feet or less). Class $G$ is regulated but completely uncontrolled. The UTM is envisioned to be designed by obeying five basic rules including UAVs do not hit each other; UAV's and manned aircraft stay away from each other; UAV operators have complete awareness of all constraints (static and dynamic); UAVs and operators are authenticated; and UAVs and operators give preferences to public safety UAVs and public safety manned aircraft (P. H. Kopardekar, 2017).

\subsection{Envisioned UTM Functionalities}

The UTM system is anticipated to provide six main services including airspace flight plan processing, operation and management, wind and weather integration, congestion management, separation management and contingency management. The UTM also needs to incorporate key technologies required for UAS operations: track and locate, detect and avoid, autonomous operation for the first/last 50 feet, weather sensing and prediction, geo-fencing, and integration of UTM service providers using a common protocol.

\subsubsection{Flight Plan Processing}

Flight planning and UAS navigation management are essential to identify the optimal route depending on the position and height of possible obstacles in the operational airspace zone (Mangiameli, Muscato, Mussumeci, \& Milazzo, 2013). In addition flight planning is an integral component of air traffic control operations, enabling efficient management of an airspace. Other ATC subsystem such as Traffic Flow Management System requires the route information from the Flight Plan Processing System (FPPS) (Paczan, Cooper, \& Zakrzewski, 2012). The FPPS should allow the UAS operator to submit their flight plan, process the flight plan and provide flight route confirmation. 


\subsubsection{Airspace Operations \& Management}

At present, UTM is envisioned to manage sUAS operations at 500 feet (ICAO, 2011; P. H. Kopardekar, 2017). Optimum utilization of an airspace depends on high performance navigation system on-board the UAV. The airspace operations management service for sUAS includes the following functions:

- Airspace design and dynamic configuration;

- Authentication of UAV and operator;

- Retrieve flight plan/trajectory;

- Route planning and re-routing;

- Dynamic geo-fencing - airspace to avoid, dynamic objects (e.g. construction equipment- crane), static objects (e.g. building, trees, lamp-post). The function should have constant update on the geo-fences to avoid;

- Terrain avoidance; and

- Conflict detection mechanism -e.g. Short-term conflict alert (STCA) Medium-term conflict alert (MTCA).

\subsubsection{Wind and Weather Integration}

The wind and weather integration service provides actual and predicted wind and weather information to the users highlighting which airspace is safe to operate and not. UAVs are more susceptible to wind due to its small size and weight, unlike helicopters and gliders. This function is a crucial safety measure for the UTM.

\subsubsection{Congestion Management}

The congestion management service ensures the balance between the demand to use the airspace and limited capacity of the airspace allocated for UAS operations. This is particularly acute in the urban areas. Potential ways to implement congestion management include creation of corridors in the airspace, altitude for direction, and crossing restrictions.

\subsubsection{Separation Management}

Separation management is underpinned by high performance (accuracy, integrity, continuity) surveillance functions to track and locate the UAVs. A number of methods are available to perform separation management for UAS: Airspace reservation- however it is not feasible to apply this method in high density airspace e.g. urban areas, and detect and avoid collision mechanism - vehicle-to-vehicle, vehicle-to-obstacle. ACAS The latter is conditioned upon availability of reliable tracking system. The service also includes sequencing and spacing of the UAVs in the airspace.

\subsubsection{Contingency Management}

The contingency management service refers to actions to counter unanticipated events that may interrupt the UTM services and UAS operations, such as large scale Global Navigation Satellite System (GNSS) or cellular system outage, breaching of geo-fences, loss of control and command (C2) of a UAV, emergency responses in the case on undesired incidents of the UAVs. UTM can be developed through these three (3) efforts, including benchmarking the current ATM system; studying and analyzing the differences between manned and unmanned aircraft and how the differences can be managed in the UTM; and studying and analyzing the characteristics of Class G airspace e.g. uncontrolled, very near to the ground and hence prone to collision with obstacles in the ground. Most importantly, the UTM requires continuous CNS support to ensure safe UAS operations and to monitor conformance to specified commands and constraints.

\section{Challenges to adopt ATM for UTM}

Air Traffic Management (ATM) is designed based on the notion of a pilot navigating an aircraft from within the aircraft. Increasing demand for Unmanned Aircraft Systems (UAS) usage and its safe integration into segregated/non-segregated airspace, on the other hand, have raised a question on the adoption of the current ATM for the UAS Traffic Management (UTM). The Procedures for Air Navigation Services - Air Traffic Management (PAN-ATM) and Procedures for Air Navigation Services - aircraft operations (PAN-OPS) rules are both developed for manned flight operations based on the notion. A 
number of challenges to implement UTM system are identified and analyzed in comparison to the ATM system.

\subsection{Piloted vs Remotely-Piloted Aircraft}

ATM was designed based on the notion of a pilot navigating an aircraft from within the aircraft. Hence providing direct control over the aircraft, particularly in terms of navigation and see and avoid operations. However, managing traffic for remotely-piloted (RPA) aircraft is a totally different ballgame. Unlike ATM, UTM is not a human centered approach. Instead, it will rely on high level of automation. Therefore, it is foreseen to be a complex endeavor with higher safety concerns. A simple example would be the see-and-avoid operation which is not applicable for the UTM.

\subsection{Procedural Control}

Current manned aircraft operations in controlled airspace are conditioned upon voice communication between pilot and ATC. This involves position reporting by pilot and altitude clearance by ATC. Since UAS pilots are not physically onboard, the means for procedural control may differ. Looking at the future demand forecast for UAS operations, voice communication may not scale to the huge demand. According to Paczan et al.(2012), the UAS could serve as a communication relay point between ATC and pilot. However, such link would be susceptible to the same link failure that occur on the vehicle Command and Control (C2) link.

\subsection{Low-Altitude Operation}

ICAO (2011) and NASA (P. H. Kopardekar, 2017) envisaged the operations of UAS airspace from 500 feet and below. This creates a huge safety concern as the operations will be very near to the ground, where humans and infrastructures are in place. In addition, UAS navigation will be exposed to beyond visual line off sights (BVLOS) situations due to high-rise buildings and infrastructures within the operational airspace range.

\subsection{Security}

The low flying operations also creates security concerns for restricted areas such as the parliament house, presidential building, royal palaces etc.

\subsection{Dense Operating Environment}

As discussed in section 1, the various potential applications of UAS indicate the dense airspace environment. According to Federal Aviation Administration (2010), it is estimated that 15000 unmanned units will be employed by 2020. Furthermore, the limited range of the Class $\mathrm{G}$ airspace will be shared with helicopters and general aviation (GA), making the limited airspace more dense. The mixed operation is also foreseen as a challenge for UTM.

\subsection{Aircraft Size and Performance}

Unlike the manned aircraft, UAV's are susceptible to severe weather such as heavy rain and thunder storms due to its small size and weight. This creates another safety concern for UTM.

\subsection{Dynamic Waypoints}

The manned aircraft have fixed waypoints from airport-to-airport. However, UAVs are meant to cater for various applications as discussed in section 1. Thus, making the routes in the class $\mathrm{G}$ airspace very complex for UAV traffic management. The level of the airspace complexity will increase the operational safety concerns.

\subsection{CNS for ATM vs CNS for UTM}


Another major challenge for UTM in comparison with the ATM, will be the choice of Communication, Navigation and Surveillance (CNS) technologies to support the system. The CNS is a backbone of any transportation traffic management system.

\subsubsection{Communication}

Communication links people and systems on the ground with the aircraft during all phases of flight. Good communication with timely and dependable availability are cornerstone of operational safety and efficiency (Syd Ali, 2013). There are two types of communication systems in place; air-ground-air and ground-ground communication. Most of the communications in ATM are air-ground based communications. The air-ground communication system relies on Very High Frequency (VHF), High Frequency (HF) and Ultra-High Frequency (UHF) analog data links (radio frequency) for en-route and terminal areas (the voice communication is also known as procedural control); and Aeronautical Mobile Satellite Service (AMSS) for oceanic and remote continental airspace (ICAO, 2000), while the groundground communication relies on VHF data link. More recent communication systems in ATM are based on digital data links such as High Frequency Data Link (HF DL), VHF Data Link Mode 4 (VDL-Mode 4), Mode-S Extended Squitter, and Universal Access Transceiver (UAT). Data link technologies enable uplink and downlink of four dimensional (4D) waypoints (latitude, longitude, altitude, time) and other data to pilots and controllers (Syd Ali, 2013). Controller Pilot Data Link Communication (CPDLC) is an example of a data link application that relies on HF DL, VDL and satellite communication (SATCOM). The ATM system operates on procedural control from day one until now. Procedural control involves delivering air traffic control services via radio communication. This include air traffic controller (ATCO) providing altitude clearance to the pilot and position reporting by the pilots to ATC. Other means of communication in ATM include duplex communication between ground systems such as radar and the aircraft, e.g. via Mode-S 1090/1030MHz. Backbone of the ATM communication is Aeronautical Telecommunication Network (ATN). ATN comprises of application entities and communication services. It enables ground elements, air-ground networks and airborne data networks interact via the International Organization for Standardization (ISO) Open System Interconnection (OSI) reference model based protocol and services interface (ICAO, 1999). Figure 1 illustrates the ATM communication environment based on the ATN. The Required Communication Performance (RCP) concept ensures that the communication system performance implemented within the ATM system is acceptably safe and reliable to operate in the ATM operational environment. The RCP concept assesses operational communication in the context of an ATM function, taking into account human interactions, procedures, and environmental characteristics (ICAO, 2006).

On the contrary, UTM communication is envisioned to have more of ground-ground communication due to the RPA nature. More on this will be discussed in section 4. 


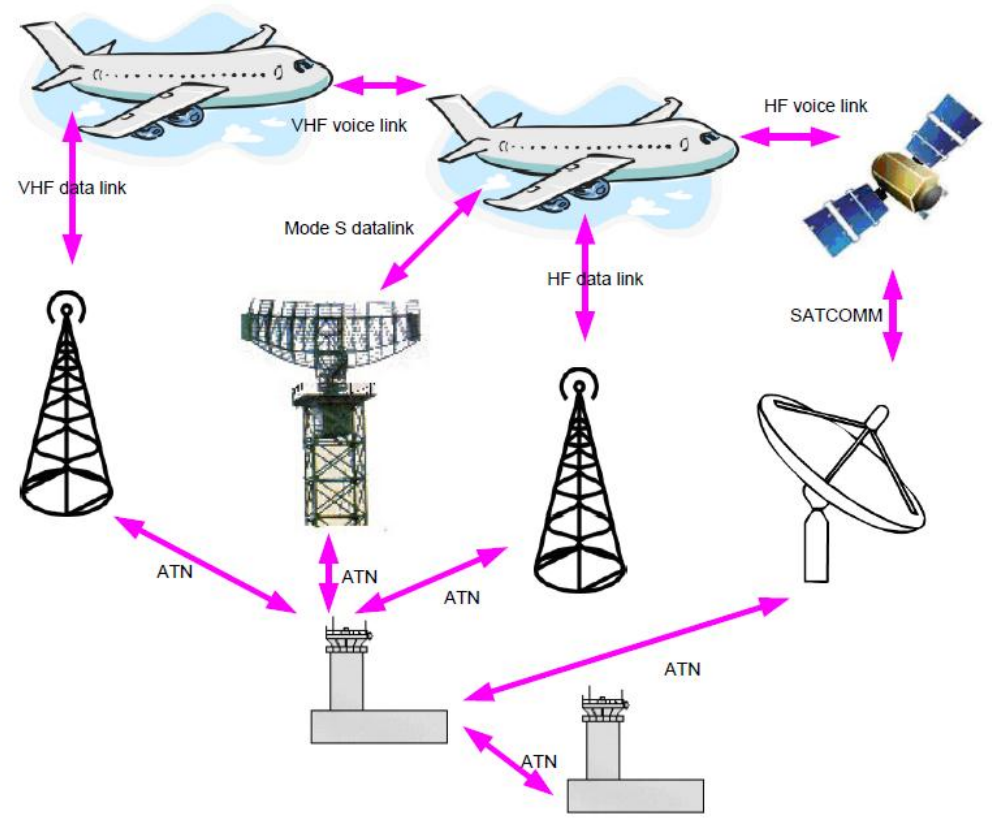

Figure 1: ATM communication environment (Syd Ali, 2013)

\subsubsection{Navigation}

Navigation refers to the ability to determine the correct state of an object then to determine the course to steer and arrive at the next desired point (ICAO, 1998a). Ground based navigation system in ATM consists of VHF Omnidirectional Radio Range/Distance Measuring Equipment (VOR/DME), NonDirectional Beacon (NDB); and Long-Range Navigation (LORAN) and Global Navigation Satellite Systems (GNSS) for area navigation (RNAV), while Instrument Landing System (ILS) and Microwave Landing System (MLS) are used for precision approaches and landing. In addition to these, ground navigation aids also include: Aeronautical Ground Lighting (AGL) system (e.g. status of runway, taxiway lighting panel), warning system (e.g. runway in use), internal lighting, meteorological equipment status, and alarming and reporting systems. Onboard navigation systems include Inertial Navigation System (INS) and the Global Positioning System (GPS) and its augmentations. Improvements in navigation in ATM systems include the progressive use of GNSS, which provides world-wide coverage. It enables the aircraft to determine its own position on-board, from the information, broadcast by the GNSS satellites. The availability of GNSS constellations (e.g. Galileo) and the further development of augmentation means (Aircraft based augmentation system (ABAS), Ground based augmentation system (GBAS), Satellite based augmentation system (SBAS)) will improve the accuracy, availability and the integrity of the navigation signal thus allowing enhanced positioning services in all phases of flight, including the airport surface. For example, surface navigation based on GBAS (using enhanced positioning based on Galileo/GPS L5) enable aircraft to navigate autonomously on the ground to the gate. In order to implement a more practical navigation specification, ICAO developed the Performance Based Navigation (PBN). PBN specifies that the aircraft Required Navigation Performance (RNP) and RNAV system performance requirements to be defined in terms of accuracy, integrity, continuity, availability and the functionalities required to operate in particular airspace supported by appropriate navigation infrastructure. Further discussion on potential navigation aids for UAS will be proposed in section 4 .

\subsubsection{Surveillance}

Surveillance acts as the "eyes" of Air Traffic Control (ATC). The capability to accurately and reliably determine the position of an aircraft at a specific time has a direct influence on the separation distances required between aircraft (i.e. separation standards), and therefore, on how efficiently a given airspace may be utilized. Depending on the type of the surveillance sensor, additional information is presented 
also such as aircraft identification and velocity. Furthermore, the surveillance function supports a number of other applications such as trajectory prediction, conflict detection and situational awareness. Requirements for an Air Traffic Control (ATC) surveillance system depend on the applications. However, no single surveillance system is capable of meeting the surveillance requirements for all phases of flight in all types of airspace with traffic conditions that vary significantly from low to high density traffic terminal areas (Syd Ali, 2013) The current ATM surveillance system in use consists of: Primary Surveillance Radar (PSR), Secondary Surveillance Radar (SSR), Monopulse Secondary Surveillance Radar (MSSR), Surface Movement Radar (SMR), Multilateration (MLAT) and Automatic Dependent Surveillance (ADS-B) systems. The Required Surveillance Performance (RSP) is a set of well-quantified surveillance performance requirements for ATM such as capacity, availability, accuracy and update rate. Any single or combination of surveillance systems meeting the targets set for the parameters is considered operationally acceptable (ICAO, 2000). Syd Ali (2013) discussed the factors or constraints on the choice of surveillance technologies for a particular airspace. These include cost, mixed aircraft equipage, geography, existing ground networking infrastructure, homogeneous surveillance infrastructure, required functionalities, equipage mandate, and airspace capacity requirements. Choice of surveillance systems for the UTM will largely depend on the geographical constraints. The constraints and proposed surveillance technology for UTM will be discussed in section 4.

\section{Proposed Architectural Framework for UTM}

Figure 2 shows proposed architectural framework for UTM. It illustrates the envisioned general components of a UTM system and their interactions. The six services described in section 2.1 resides in the UTM server. The server interacts with Air Traffic Control (ATC) Centre to obtain essential Aeronautical Information comprising of Aeronautical Information Publication (AIP), Aeronautical Information Circular (AIC), Notice to Airmen (NOTAM)-alerts pilot of any hazards at a specific location, and weather information. The UTM server is also integrated to a surveillance data processing system (SDPS) that provides UAVs and other cooperative aircraft including GA's real-time positioning information in a particular control area. Air Traffic Controllers (ATCO) at the ATC Centre is also able to communicate with the UTM Manager and the UAS Pilots residing at the Remote Pilot Stations for e.g. to convey information on potential conflict between an UAV and manned aircraft or controlled airspace intrusion by an UAV. The ATC Centre will also be able to access the services in the UTM server via a UTM client.

The UTM server is accessed by the UAS Pilots residing at the Remote Pilot Station via a UTM Client (to submit flight plan; obtain approved flight trajectory; attain traffic situational awareness, wind and weather information; to receive conflict alerts -via STCA, MTCA; and to obtain updated geo-fencing information). UAS Pilots are also able to communicate with each other via a dedicated communication link.

Finally, the interaction between the UAS Pilot and UAVs to perform command and control (C2) of the UAV for safe and smooth maneuvering. 


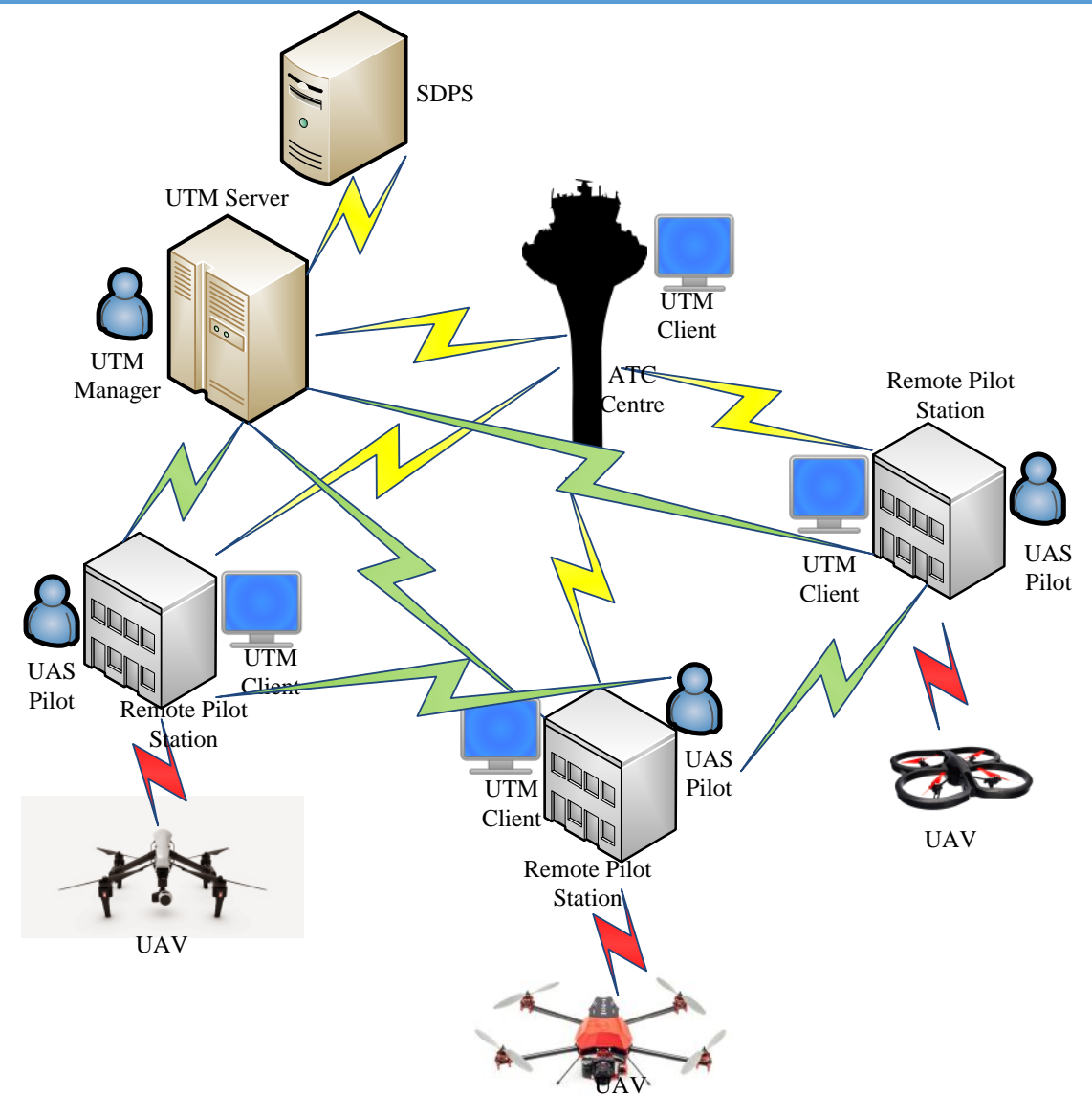

Figure 2: UTM Architectural Framework

The aim of Communication, Navigation and Surveillance (CNS) technologies is to provide a comprehensive support to enable provision of traffic services for UAS operations in the limited airspace capacity with associated safety and efficiency, providing the desired routes to the airspace users, and homogenizing the use of equipment in the airspace. CNS for UTM is underpinned by a high level of automation which reduces the dependency on the human and eliminates the constraints to optimize the airspace capacity.

Table 1 provides the proposed communication technologies for each component interactions illustrated in Figure 2. As mentioned in section 3.8.1, the mode of communication in UTM will mostly be ground-to-ground communication due to the RPA nature of UAS. It is also envisioned that the communication will be based on data links. Based on the sUAS operation nature, real-time communication tool apps such as Skype (support voice and data over IP) can be used for the groundto-ground communication, particularly between the ATCo-UTM Manager, ATCo-UAS Pilot, UAS PilotUAS Pilot and UAS Pilot-UTM Manager. All registered UAVs should have a Skype_ID with the app residing in the UTM Server and accessed via the UTM clients.

Table 1: Proposed Communication Technologies

\begin{tabular}{ll}
\hline \multicolumn{1}{c}{ UTM Components Interactions } & \multicolumn{1}{c}{ Communication Technologies } \\
\hline UTM Server-ATC Centre & TCP/IP using Ethernet, Leased line or Fiber \\
UTM Server-SDPS & TCP/IP using Ethernet, Leased line or Fiber \\
ATCo-UTM Manager & VOIP and TCP/IP using Ethernet, Leased line or \\
& Fiber \\
\hline
\end{tabular}


THE 22ND AIR TRANSPORT RESEARCH SOCIETY (ATRS) WORLD CONFERENCE AT COEX,

SEOUL, SOUTH KOREA FROM JULY 2-5, 2018

\begin{tabular}{ll}
\hline \multicolumn{1}{c}{ UTM Components Interactions } & \multicolumn{1}{c}{ Communication Technologies } \\
\hline ATCo-UAS Pilot & VOIP and TCP/IP using Ethernet, Leased line or \\
& Fiber \\
ATCo-UTM Client & TCP/IP using Ethernet, Leased line or Fiber \\
UAS Pilot-UAS Pilot & VOIP and TCP/IP using Ethernet, Leased line or \\
& Fiber \\
UAS Pilot-UTM Client & TCP/IP using Ethernet, Leased line or Fiber \\
UAS Pilot-UAV & C2 Frequency Links with data encryption \\
& capabilities \\
\hline
\end{tabular}

High performance (accuracy, integrity, reliability and continuity) surveillance system is the key to the UTM system. The surveillance data is used as input for separation management, conflict detection (STCA, MTCA), airspace capacity management, detect and avoid collision mechanism, and also UAV take-off and landing operations. The data also provides airspace situational awareness to the UTM Manager for traffic control and UAS Pilots for safe UAV navigation. Choice of surveillance technology for UTM largely depends on geographical area and its surface. Due to UAS's low level operations, radar is considered as unsuitable due to its high radiation level, particularly in the urban areas. Automatic Dependent Surveillance Broadcast (ADS-B) system is one of the best solution for UAS surveillance. However, its sole dependence on Global Satellite Navigation System (GNSS) introduces line-of-sight problems especially in the urban areas where high rise buildings may interrupt the GNSS signal and hence limits ADS-B coverage. This limitation will definitely affect the take-off and landing operations particularly in the urban area. Unlike the manned aircraft operation whereby surface navigation based on GBAS enable aircraft to navigate autonomously on the ground to the gate, is not applicable to sUAS operation in the urban setting. On the hand, concept of Multilateration (MLAT) / Wide Area Multilateration (WAM) requiring at least three WAM ground sensors to derive aircraft positioning information, is foreseen as one of the limitation of this technology in terms of its adaptability to the urban setting. Even though MLAT system is way more effective than Surface Movement Radar (SMR) for airport surface surveillance, it is not as effective for sUAS in the urban area. MLAT/WAM system relies on the Mode-S signal which is also vulnerable to line-of-sight and interference issues. A better option for the second layer surveillance would be cellular technology such as $4 G$ and LTE. 4G is the fourth generation of mobile data technology, as defined by the radio sector of the International Telecommunication Union (ITU-R). LTE stands for "Long Term Evolution" and applies more generally to the idea of improving wireless broadband speeds to meet increasing demand. The advantages of the cellular technology include established existing infrastructures particularly in the urban area and absence of line-of-sight and interference problems. In addition, it would allow uninterrupted service in the low altitude particularly for the take-off and landing operations. However, ADS-B is proposed as the primary surveillance layer enabling ultra-low cost and low power system to allow fully cooperative traffic between both sUAS and manned aircraft (GA) in the low altitude class G airspace. The ADS-B ground receivers are scalable and can be installed on buildings or any existing infrastructures. The proposed solution for sUAS surveillance has high level of robustness. In the case of GNSS total outage, the UAVs can still be tracked via the cellular system.

Navigation for UTM will be entirely a different ballgame. Unfortunately, the ground based navigation systems used in the ATM (discussed in section 3.8.2) are not applicable for UTM. The UAS does not have a specific landing area like the manned aircraft. However, the onboard navigation systems used in the ATM can be potentially deployed for the UTM. It is proposed that, with the onboard navigation systems, an app like Waze (Waze Mobile, 2018) integrated to a Geographical Information System (GIS) would be a good solution for sUAS navigation in UTM.

\section{Conclusion}

Based on the findings in this work, it is clear that there are more safety concerns in the UTM system in comparison to the ATM system. It is feasible to adopt some part of the ATM operational concept. However, it has to be meticulously studied and modified to mitigate the challenges in UTM identified in this work (refer to section 3). The main safety concern arose from the fact that sUAS will not be 
operated by pilots onboard the aircraft; and their operations in low altitude airspace, exposed to humans and infrastructures in the ground. This work (i) defined the UTM system and described its envisioned functionalities; (ii) performed an exploratory research to identify the distinctions between a manned and an unmanned flight operations and consequently the possible challenges to implement UTM with regard to the ATM; (iii) determined potential Communication, Navigation and Surveillance (CNS) technologies to support the UTM system; and proposed an architectural framework for UTM based on the findings. The proposed solution in section 4 reckons a comprehensive proof-of-concept (POC) by developing case studies in the urban setting in collaboration with key players from the industry.

\section{References}

Amazon Prime Air. (2015a). Determining safe access with a best-equipped, best-served model for small unmanned aircraft systems. In NASA (Ed.).

Amazon Prime Air. (2015b). Revising the airspace model for the safe integration of small unmanned aircraft systems. In NASA (Ed.).

Federal Aviation Administration. (2010). FAA Aerospace Forecast Fiscal Years 2010-2030.

Foina, A. G., Krainer, C., \& Sengupta, R. (2015). An unmanned aerial traffic management solution for cities using an air parcel model. Paper presented at the International Conference on Unmanned Aircraft Systems (ICUAS).

Google Inc. (2015). Google UAS Airspace System Overview. In NASA (Ed.).

ICAO. (1999). Manual of Technical Provisions for the Aeronautical Telecommunication Network. Montreal, Canada: International Civil Aviation Organization.

ICAO. (2000). National Plan for CNS/ATM Systems (Vol. DOC 278AN/164): International Civil Aviation Organization.

ICAO. (2006). Manual on Required Communication Performance (RCP) (Vol. Doc 9869 AN/462). Montreal, Canada: International Civil Aviation Organization.

ICAO. (2011). Unmanned Aircraft Systems (UAS). In I. C. A. Organization (Ed.), Circular 328AN/190: International Civil Aviation Organization

International Civil Aviation Organization. (2005). Global Air Traffic Management Operational Concept Doc 9854 AN/458.

Jiang, T., Geller, J., Ni, D., \& Collura, J. (2017). Unmanned Aircraft System traffic management: concept of operation and system architecture. International journal of transportation science and technology, 5(3), 123-135.

Kopardekar, P. (2016). Unmanned Aircraft System Traffic Management System. In Google (Ed.), Talks at Google.

Kopardekar, P., Rios, J., Prevot, T., Johnson, M., \& Jung, J., \& Robinson, J. (2016). Unmanned aircraft system traffic management (UTM) concept of operations AlAA Aviation Forum.

Kopardekar, P. H. (Producer). (2017). Unmanned Aircraft Systems Traffic Management (UTM) Safely Enabling UAS Operations in Low-Altitude Airspace. Retrieved from https://ntrs.nasa.gov/archive/nasa/casi.ntrs.nasa.gov/20170001573.pdf

Low, K. H., Gan, L., \& Mao, S. (2014). A Preliminary Study in Managing Safe and Efficient LowAltitude Unmanned Aircraft System Operations in a Densely Built-up Urban Environment. Air Traffic Management Research Institute, School of Mechanical and Aerospace Engineering Nanyang Technological University.

Mangiameli, M., Muscato, G., Mussumeci, G., \& Milazzo, C. (2013). A GIS application for UAV flight planning. Paper presented at the IFAC. 
Paczan, N. M., Cooper, J., \& Zakrzewski, E. (2012). Integrating unmanned aircraft into NextGen automation systems. Paper presented at the Digital Avionics Systems Conference (DASC) IEEE/AIAA 31st.

Pathiyil, L., Low, K. H., Soon, B. H., \& Mao, S. (2016). Enabling Safe Operations of Unmanned Aircraft Systems in an Urban Environment: A Preliminary Study. Paper presented at the International Symposium on Enhanced Solutions for Aircraft and Vehicle Surveillance Applications (ESAVS).

Prevot, T., Homola, J., \& Mercer, J. (2016). From rural to urban environments: human/systems simulation research for low altitude UAS Traffic Management (UTM. Paper presented at the 16th AIAA Aviation Technology, Integration, and Operations Conference.

Prevot, T., Rios, J., Kopardekar, P., Robinson III, J. E., Johnson, M., \& Jung, J. (2016). UAS traffic management (UTM) concept of operations to safely enable low altitude flight operations. Paper presented at the 16th AIAA Aviation Technology, Integration, and Operations Conference.

Ren, L., Castillo-Effen, M., Yu, H., Yoon, Y., Nakamura, T., Johnson, E. N., \& 2017lppolito, C. A. (2017). Small unmanned aircraft system (sUAS) trajectory modeling in support of UAS traffic management (UTM). Paper presented at the 17th AIAA Aviation Technology, Integration, and Operations Conference.

Syd Ali, B. (2013). A safety assessment framework for Automatic Dependent Surveillance Broadcast (ADS-B) and its potential impact on aviation safety. (PhD), Imperial College London, England, United Kingdom.

Waze Mobile. (2018). Waze. Retrieved 1 May, 2018, from https://www.waze.com/en-GB/ 